\title{
Study of Device State Recognition Algorithm Based on Improved YOLOv3
}

\author{
Xiansong Bao, Gu Hao*, Zhang Fan
}

Anhui Nanrui Jiyuan Power Grid Technology Co., Ltd., Hefei, Anhui Province, China

DOI: $10.36348 /$ sjet.2020.v05i08.001

| Received: 07.08.2020 | Accepted: 14.08.2020 | Published: 29.08.2020

*Corresponding author: Gu Hao

\section{Abstract}

In view of the timeliness and accuracy of traditional state recognition algorithms, this paper proposes an improvement measure for foreground segmentation and target recognition. Foreground segmentation is to model the background information in the scene before recognizing the image, to separate the foreground target from the scene, at the same time to reduce the impact of noise, shadow and other environmental changes as much as possible, and then segment the target through a sliding window strategy. Target recognition is improved with reference to the PRN network and anchorboxes mechanism, and a more advanced clustering k-means++ algorithm is applied. The method has low error rate, high signalto-noise ratio and fast processing speed. Finally, the proposed improved algorithm is applied to device status recognition, which shows the advanced nature of the algorithm.

Keywords: background model; image recognition; image segmentation; clustering algorithm; algorithm optimization.

Copyright @ 2020: This is an open-access article distributed under the terms of the Creative Commons Attribution license which permits unrestricted use, distribution, and reproduction in any medium for non-commercial use (NonCommercial, or CC-BY-NC) provided the original author and source are credited.

\section{INTRODUCTION}

In substations, real-time monitoring of equipment status is required to prevent accidents caused by sudden changes of it. The actual state of the pressure plate and knife gate needs to be accurate in the daily production and maintenance of the substation [1]. Video surveillance has been fully implemented in some modern substations, thus, the control center can observe the real-time status of the production site, including status images such as pressure plates and knife gates.

This paper takes the substation protection cabinet pressure plate as the research object, and uses the foreground segmentation and target recognition technology to process the image. Then, after algorithm optimization, the YOLOv3 method is used to train the equipment state detection model.

\section{YOLOv3 Algorithm Model Model Characteristics}

Since the traditional meter recognition algorithm uses only image processing algorithms, the accuracy is not high [2]. Deep learning target detection technology has been widely used in recent years, and as one of the representative models of target detection, the biggest feature of the YOLOv3 algorithm models is its fast speed and high accuracy, so it is the first choice for real-time image processing.

The characteristics of the YOLOv3 algorithm model are: drawing on the residual network structure, forming a deeper network level and multi-scale detection, which improves the detection effect of mAP and small objects [3]. If COCO mAP50 is used as the evaluation index, the performance of YOLOv3 is quite amazing. In the case of equivalent accuracy, the speed of YOLOv3 is 3 or 4 times that of other models. 


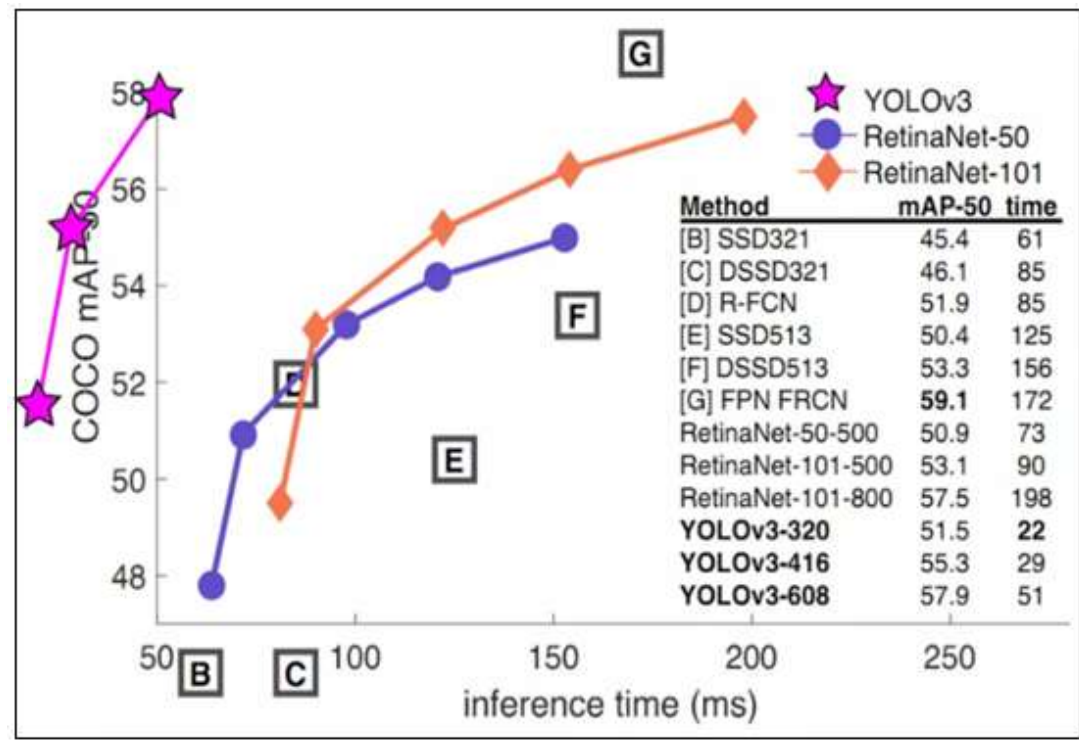

Fig-1: Comparison of YOLOv3 algorithm models

\section{Model Structure}

The structure of the YOLOv3 algorithm model is: in simple terms, in terms of basic image feature extraction [4], YOLO3 uses a network structure called
Darknet-53 (containing 53 convolutional layers), which draws on the practice of residual networks and sets up shortcut connections between some layers.

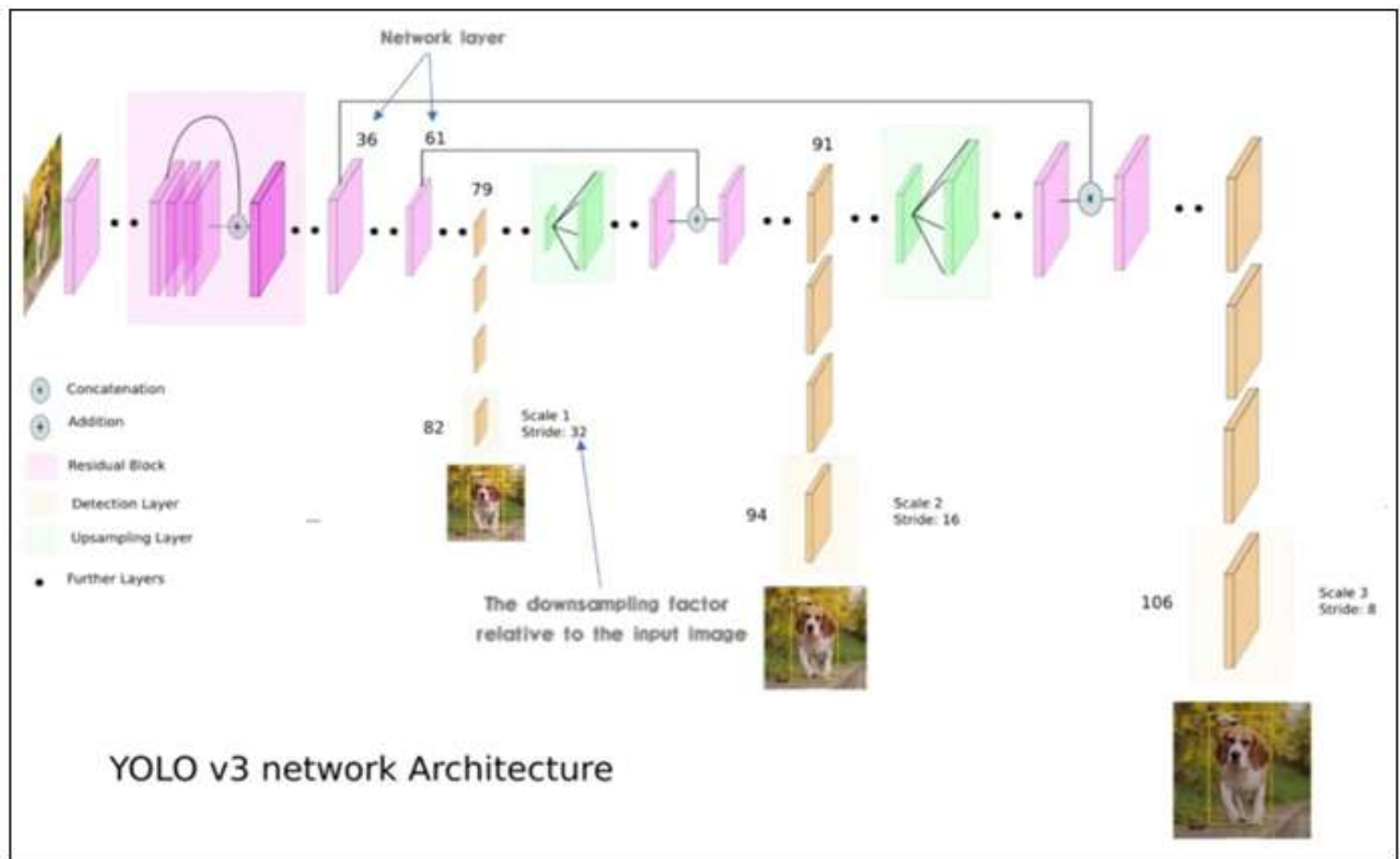

Fig-2: YOLOv3 algorithm model structure diagram

The network structure of YOLOV3 is divided into the following modules [5]. The first layer is the convolution layer, where we put a picture. The number of channels of the image is 3 , and the pixels are $416 * 416$. We use 32 convolutional layers to extract image features [6]. The size of each convolution kernel is $3 * 3$ and the step size is 1 . After the convolution operation, a $416 * 416$ 32-channel feature map is obtained.
The second layer is the res layer, which is derived from the nearest layer. In order to solve the phenomenon of network gradient dispersion or gradient explosion [7], it is proposed to change the layer-bylayer training of deep neural network to step-by-step training, and divide the deep neural network into several sub-segments, each of which contains relatively shallow content. 
The third part is dark -53: a total of 53 convolutional layers from layer 0 to layer 74 , the rest are reslayers. At the same time, in the case of better performance, its efficiency is 1.5 times that of resnet101. Its efficiency is almost twice that of resnet-152, the same effect as resnet-152. The last layer is the feature interaction layer of the yolo network. It is divided into three scales. After the training, we will get the target box and category probability [8]. By setting the threshold, we can predict the target.

\section{YOLOv3 algorithm model improvement scheme Foreground Segmentation}

For the image collected by the camera, in order to reduce the interference of the background area on the identification target, we model the background information in the monitoring scene before identifying the image. The goal is to separate the foreground target from the scene, and at the same time reduce the impact of noise, shadows and other environmental changes as much as possible, and then segment the target we need through a sliding window strategy to facilitate later identification.

\section{Target Recognition}

The report algorithm contains two parts of optimization. Part of it is based on the detailed understanding of the YOLOv3 operation mode, using the anchorboxes mechanism used by the PRN network and Box regression to improve. Its role is to generate object proposals in the network, generate Bounding Boxes through screening, and obtain new anchor boxes through more advanced clustering algorithms. Specifically, a more advanced clustering k-means++ algorithm is applied, and the loss function is calculated with IoU.

The original K-means algorithm initially randomly selected $\mathrm{K}$ points in the data set as the cluster center. However, K-means++ selects K cluster centers according to the following ideas: Assuming that $\mathrm{n}$ initial cluster centers have been selected $(0<\mathrm{n}<\mathrm{K})$, when selecting the $n+1$ th cluster center: The point farther away from the current $\mathrm{n}$ cluster centers will have a higher probability to be selected as the $\mathrm{n}+1$ th cluster center. When selecting the first cluster center $(n=1)$, the random method is also adopted. It is better for the cluster centers to be farther away from each other. Although this improvement is intuitive and simple, it is very effective.

\section{EXPERIMENT AND ANALYSIS}

\section{Testing process}

The main function of the opening and closing status module is to analyze the operating status of the electrical equipment through automatic detection in complex scenarios. Detection identification set, data calibration, training package, front-end application. The process is shown below:

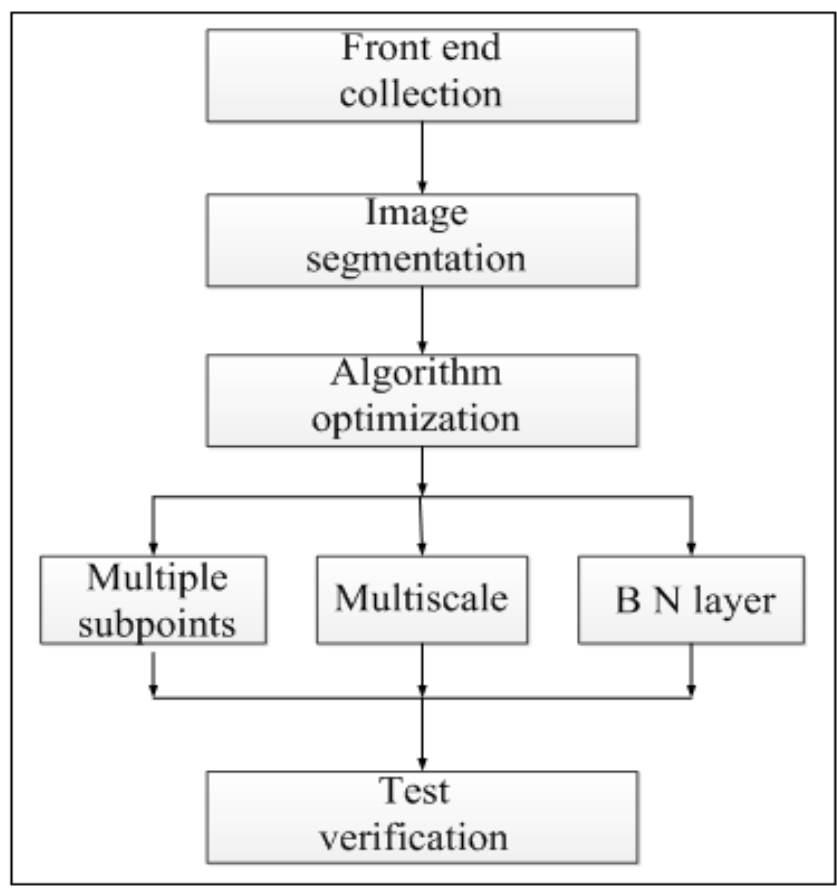

Fig-3: Flowchart of target recognition

\begin{abstract}
Algorithm Comparison Analysis
The experiment selected about 8,000 sample images collected from the substation site, with a total number of about 20,000 targets, including 6,800 training sets and 1,200 test sets. Based on the improved
\end{abstract}

YOLOv3 algorithm model for identification and analysis, a comprehensive evaluation was carried out from the officially recognized evaluation standards (accuracy, false detection rate, and missed detection rate). 
Table-1: Data before improvement

\begin{tabular}{|c|c|}
\hline Accuracy & 0.85 \\
\hline False detection rate & 0.05 \\
\hline Missed detection rate & 0.10 \\
\hline
\end{tabular}

Table-2: Data after improvement

\begin{tabular}{|c|c|}
\hline Accuracy & 0.91 \\
\hline False detection rate & 0.02 \\
\hline False detection rate & 0.07 \\
\hline
\end{tabular}

For the same test set, the accuracy, false detection and missed detection rate of the algorithm are improved by $6 \%, 3 \%$, and $3 \%$ respectively compared with the original algorithm. Especially for the situation where the target is difficult to distinguish in a complex background, it has better promotion and improvement.

\section{CONCLUSION}

Based on YOLOv3, this paper proposes an improvement measure for the device state recognition algorithm. Foreground segmentation is better than the fuzzy C-means method and the OTSU method. The segmentation effect of this method is better, especially when image brightness is uneven and contrast is low, the segmentation error is small. However, the operation efficiency needs to be improved in the future.

\section{REFERENCES}

1. Li, D., Liu, M., \& Guo, S. (2016). Application of image segmentation based on improved K_means algorithm. Computer Knowledge and Technology.
12(8):166-168

2. Redmon, J., Divvala, S., Girshick, R., \& Farhadi, A. (2016). You only look once: Unified, real-time object detection. In Proceedings of the IEEE conference on computer vision and pattern recognition (pp. 779-788)

3. Huang, L., Chang, J., Yang, F., Li, Y., \& Niu, X. (2020). Anomaly detection method for power information system based on improved k-means. Journal of Shenzhen University (Science and Technology Edition). 37(2):214-220.

4. Zhang, L., \& Mou, X. (2018). Chinese text clustering algorithm based on Canopy+K-means. Library Forum. 38(6):113-119.

5. Jiang, B., Wu, Q., Yin, X., Wu, D., Song, H., \& He, D. (2019). FLYOLOv3 deep learning for key parts of dairy cow body detection. Computers and Electronics in Agriculture, 166, 104982.

6. Science - Applied Sciences. (2019). Research Results from Jiangsu University Update Understanding of Applied Sciences (Application Research of Improved YOLO V3 Algorithm in PCB Electronic Component Detection). Science Letter.

7. Shuo, L., Xuan, C., \& Rui, F. (2019). YOLOv3 network based on improved loss function. Computer Systems and Applications, 28(2), 1-7.

8. Tan, J. (2018). Research on an improved yolov3 target recognition algorithm. Huazhong University of Science and Technology, 1-66. 